\title{
Detection of Target ssDNA Using a Microfabricated Hall Magnetometer with Correlated Optical Readout
}

\author{
Steven M. Hira, ${ }^{1}$ Khaled Aledealat, ${ }^{2}$ Kan-Sheng Chen, ${ }^{2}$ Mark Field, ${ }^{3}$ Gerard J. Sullivan, ${ }^{3}$ \\ P. Bryant Chase, ${ }^{4,5}$ Peng Xiong, ${ }^{2,5}$ Stephan von Molnár, ${ }^{2,5}$ and Geoffrey F. Strouse ${ }^{1,5}$
}

\author{
${ }^{1}$ Department of Chemistry and Biochemistry, The Florida State University, Tallahassee, FL 32306-4390, USA \\ ${ }^{2}$ Physics Department, The Florida State University, Tallahassee, Fl 32306-4350, USA \\ ${ }^{3}$ Teledyne Scientific Company LLC, Thousand Oaks, CA 90360, USA \\ ${ }^{4}$ Department of Biological Science, The Florida State University, Tallahassee, FL 32306, USA \\ ${ }^{5}$ Integrative NanoScience Institute, The Florida State University, Tallahassee, FL 32306, USA
}

Correspondence should be addressed to Geoffrey F. Strouse, strouse@chem.fsu.edu

Received 8 July 2011; Accepted 20 August 2011

Academic Editor: Alf Månsson

Copyright ( $\odot 2012$ Steven M. Hira et al. This is an open access article distributed under the Creative Commons Attribution License, which permits unrestricted use, distribution, and reproduction in any medium, provided the original work is properly cited.

\begin{abstract}
Sensing biological agents at the genomic level, while enhancing the response time for biodetection over commonly used, opticsbased techniques such as nucleic acid microarrays or enzyme-linked immunosorbent assays (ELISAs), is an important criterion for new biosensors. Here, we describe the successful detection of a 35-base, single-strand nucleic acid target by Hall-based magnetic transduction as a mimic for pathogenic DNA target detection. The detection platform has low background, large signal amplification following target binding and can discriminate a single, $350 \mathrm{~nm}$ superparamagnetic bead labeled with DNA. Detection of the target sequence was demonstrated at $364 \mathrm{pM}(<2$ target DNA strands per bead) target DNA in the presence of $36 \mu \mathrm{M}$ nontarget (noncomplementary) DNA ( $<10$ ppm target DNA) using optical microscopy detection on a GaAs Hall mimic. The use of Hall magnetometers as magnetic transduction biosensors holds promise for multiplexing applications that can greatly improve point-of-care (POC) diagnostics and subsequent medical care.
\end{abstract}

\section{Introduction}

The ability to detect and discriminate specific nucleic acid sequences within a biological mixture has implications for genome sequencing and single-nucleotide polymorphism (SNP) detection, biowarfare target detection, and the development of an efficient point-of-care (POC) device for pathogen identification [1-6]. Through the integration of biology with nanotechnology, a detection platform utilizing magnetic transduction can capitalize on the high biological specificity of DNA base pairing, the scalability of nanotechnology, the selectivity of self-assembled monolayer technology, and the sensitivity of magnetic transduction $[7,8]$. Coupling the extreme sensitivity of Hall-based magnetic detection, which operates over a wide magnetic field and temperature range, with the versatility and specificity of DNA base pairing can allow the realization of a new biological detection strategy that will improve POC diagnostics and subsequent medical treatment.
In this paper the detection of a 35-base pair DNA target sequence is demonstrated at the single-bead level on a Hall magnetometer biosensor. The biosensor is able to identify a single-bead bound to target DNA ( 35 bases) and is amenable to the discrimination of DNA at the $364 \mathrm{pM}$ concentration in a background of $36 \mu \mathrm{M}$ noncomplementary DNA ( $<10 \mathrm{ppm})$. The detection strategy utilizes three-strand DNA annealing to colocalize a superparamagnetic (SPM) bead labeled probe strand, a label-free target strand, and a receptor strand at the surface of the Hall device. Localization of the SPM bead on the surface of the Hall cross' active area through annealing of all three DNA strands induces a voltage change in the Hall junction due to a change in the local magnetic field. This study demonstrates the effective use of an optical/magnetic bead detection platform to measure DNA at the picomolar (pM) level in the presence of $\mu \mathrm{M}$ extraneous DNA. At the concentrations of DNA used in the mimic, the device platform can be optimized for clinical 
translation. Development of single-nucleotide mismatch and real-world pathogen samples are underway, but are beyond the scope of the current study.

Many biosensors [9-24] still suffer from limitations in stability, portability, sensitivity, and selectivity. Traditional ELISA based sensor platforms are sensitive at the pM level and require 1-2 days for detection of a protein target. GMR sensors, which are recent additions to the biosensor field, can detect at the pM or femtomolar (fM) levels if magnetically assisted [25]. Optics-based sensors, whether colorimetric or using FRET assays, allow detection at the attomolar (aM) to nanomolar (nM) level. A novel approach utilized in some optics-based biosensors is the use of three-strand DNA annealing to produce an optical response that is directly proportional to the annealing event. The use of threestrand ssDNA annealing strategies has been investigated for biological target detection for the last 15 years and has been shown to increase overall sensitivity. Mirkin et al. first used the controlled assembly and aggregation of DNA labeled Au nanoparticles in solution as a colorimetric sensor [26]. Years later Taton et al. [14] utilized the tethering of DNA-coated Au nanoparticles to DNA-coated surfaces using an unlabeled target sequence for Ag-amplified colorimetric detection with single-nucleotide mismatch sensitivity. The technology has evolved further and been shown to detect $\sim 6 \times 10^{6}$ copies of genomic DNA using Ag-amplified scanometric detection on a commercial platform $[27,28]$. In addition to the assembly of Au nanoparticles, the assembly of Ag nanoparticles onto smooth metal films using three-strand DNA assembly has been demonstrated for surface-enhanced Raman spectroscopic detection of DNA sequences [29]. Optical methods focused on fluorescence blotting assays have reached aM sensitivities [23], while methods employing energy transfer detection of the three-strand assembly allow nM pathogen DNA detection [20]. Despite these applications of multisequence DNA assembly in the literature, the use of three-strand assemblies for magnetic detection has only been suggested and remains underutilized [30].

Sensing technologies based on magnetic transduction, whether Hall magnetometry or giant magnetoresistive (GMR), circumvent many of the limitations of classical sensor designs since they exhibit low sensitivity to the surrounding biological matrix of samples, can be mass produced, and, if configured properly, can offer dynamic detection in a microfabricated scalable platform [31, 32]. Magnetic transduction-based sensing technologies cover a wide range of methods including GMR sensors through the use of spin valves [25, 33-37] or bead array counters (BARCs) [38, 39] and Hall-based sensors [40-45]. Already, examples of GMR devices have demonstrated detection of matrix-insensitive protein assays at the $\mathrm{fM}$ level using a magnetic transductionbased device [37] and aM level by adding additional magnetic beads to amplify the signal [46]. The use of Hall magnetometry for DNA biosensing could represent the next generation for magnetic transduction-based devices, since a Hall junction is a 4-point probe device (current, voltage), scalable down to the nanoscale, can be mass produced using standard lithographic and fabrication methods, displays a linear response through a wide range of magnetic fields [47] with minimal influence of temperature [48] (which varies to optimize DNA annealing), and can operate at high frequency allowing for phase-sensitive detection of the transient fields associated with SPM nanoscale beads. Hall biosensors may thus offer a useful alternative to exclusively fluorescencebased microarray technologies.

\section{Materials and Methods}

2.1. Substrate Fabrication and Passivation. The $1 \mu \mathrm{m}^{2}$ Hall junction is fabricated into the surface of an epitaxially grown heterostructure consisting of a GaAs substrate containing an InAs quantum well core, and $\mathrm{SiO}_{2}(60 \mathrm{~nm})$ was sputtered onto the device followed by a layer of $\mathrm{Ti}(5 \mathrm{~nm})$ and deposition of $3 \mu \mathrm{m}$ gold pads ( $20 \mathrm{~nm}$ thick) directly over the protected Hall junction. Registry of the gold pad was accomplished by photolithography using alignment markers in the photomask. Mimic microarrays ( $3 \mu \mathrm{m}$ diameter circles and $2 \mu \mathrm{m} \times 4 \mu \mathrm{m}$ rectangular gold patterns) were fabricated onto the $\langle 100\rangle$ face of a single-crystal GaAs wafer. The substrates were cleaned prior to use for $1 \mathrm{~min}$ at low power in oxygen plasma (Harrick Plasma PDC-001). The substrates were rinsed with absolute ethanol for $1 \mathrm{~min}$ and dried under a constant stream of nitrogen gas, and the $\mathrm{SiO}_{2}$ surface was passivated by 2-[methoxy(polyethyleneoxy)propyl]trimethoxysilane (Gelest) [49].

\subsection{DNA Immobilization and Hybridization. Synthetic DNA} oligonucleotides were commercially synthesized (Midland Certified Reagent Company). The two-strand DNA system consisted of a $5^{\prime}$ disulfide modified complementary receptor sequence $5^{\prime}$-/RSSR/-GAC TAC TCT ATC GGC AGC TAA GAT TGT CAC AGT CG-3' , a 5' disulfide modified noncomplementary receptor sequence $5^{\prime}$-/RSSR/-CGA CTG TGA CAA TCT TAG CTG CCG ATA GAG TAG TC-3', and a $5^{\prime}$ modified biotinylated probe sequence with an internal fluorescein dT 5'-/BIOTIN/-CGA C-/iFLUORdT/-G TGA CAA TCT TAG CTG CCG ATA GAG TAG TC-3' . The threestrand DNA system consisted of a probe sequence $5^{\prime}$-TCA TTC ACA CAC -/iFLUORdT/-CG/3BIOTIN/-3' labeled with an internal fluorescein $\mathrm{dT}$ and biotin, receptor sequence $5^{\prime}$ /RSSR/GTC TTG TCT CCT GTC AGC TA-3' with a disulfide modifier, a 35-base unmodified target sequence $5^{\prime}$-CGA GTG TGT GAA TGA TAG CTG ACA GGA GAC AAG AC$3^{\prime}$, and a 35-base unmodified nontarget control sequence $5^{\prime}$ GTC TAA GAG TGT CCT GGC TAT GAT CCG TGA GTA TG-3'. The lyophilized DNA was buffer-exchanged using an NAP-V size exclusion column (GE Healthcare) equilibrated with $20 \mathrm{mM}$ sodium phosphate buffer, $50 \mathrm{mM} \mathrm{NaCl} \mathrm{pH} \mathrm{7.0.}$

The receptor DNA (disulfide not previously reduced) was incubated on top of the device in the form of a $50 \mu \mathrm{L}$ droplet at a DNA concentration of $9 \mu \mathrm{M}$ for $6 \mathrm{hrs}$ in an enclosed incubation chamber. The incubation chamber also contains a supersaturated $\mathrm{NaCl}$ solution to maintain constant humidity within the enclosed chamber. The device was immersed in $5 \mathrm{~mL}$ of $18.2 \mathrm{M} \Omega-\mathrm{cm}$ nanopure $\mathrm{H}_{2} \mathrm{O}$ (Barnstead) containing 0.1\% Tween-20 (v/v), twice in $5 \mathrm{~mL}$ of $18.2 \mathrm{M} \Omega-\mathrm{cm}$ nanopure $\mathrm{H}_{2} \mathrm{O}$ to rinse and remove unbound 
DNA, and dried under a constant stream of nitrogen gas. The reporter DNA was bioconjugated to the SPM nanobead ( $350 \mathrm{~nm}$ mean size, Bangs Laboratories) through a biotinstreptavidin linkage at $30^{\circ} \mathrm{C}$ for $1 \mathrm{hr}$. The DNA-nanobead conjugate was purified away from free DNA using magnetic separation and washing the sample 5 times with $20 \mathrm{mM}$ sodium phosphate buffer, $300 \mathrm{mM} \mathrm{NaCl}, \mathrm{pH}$ 7.0. The threestrand DNA strategy included a preconjugation step of the target DNA to the probe DNA-SPM conjugate at $80^{\circ} \mathrm{C}$ and was allowed to slowly cool to room temperature over $1 \mathrm{hr}$. Unbound nucleic acid was removed by magnetic separation. The hybridization assay was carried out by incubating a $25 \mu \mathrm{L}$ droplet of target biotinylated DNA $(7 \mu \mathrm{M})$ bound to streptavidin-coated SPM beads for $2 \mathrm{hrs}$ in an enclosed incubation chamber containing a super saturated $\mathrm{NaCl}$ solution. The device was washed once in $5 \mathrm{~mL}$ of $20 \mathrm{mM}$ sodium phosphate buffer with $300 \mathrm{mM} \mathrm{NaCl}$ at $\mathrm{pH} 7.0$ containing $0.1 \%$ Tween-20 (v/v), twice in $5 \mathrm{~mL}$ of $20 \mathrm{mM}$ sodium phosphate buffer with $300 \mathrm{mM} \mathrm{NaCl}$ at $\mathrm{pH} 7.0$, stored in $20 \mathrm{mM}$ phosphate buffer with $300 \mathrm{mM} \mathrm{NaCl}$ at $\mathrm{pH}$ 7.0 , and protected from ambient light.

2.3. Microscopy. Fluorescence microscopy was carried out on an inverted Nikon TE2000-E2 Eclipse microscope (Nikon Instruments Inc.) equipped with a Nikon CFI Plan Apochromat 40x objective (NA 0.95, $0.14 \mathrm{~mm}$ WD). Widefield imaging of the substrates utilized an EXFO E-Cite illumination source and a FITC filter (Chroma, ex: 480/30, DCLP: 505, em: 535/40). Images were acquired on a Photometrics Coolsnap $\mathrm{HQ}_{2}$ CCD camera. Bright-field overlays utilized differential interference contrast (DIC) to observe the differences in the index of refraction of the samples. The data were analyzed using Nikon NIS Elements software. Scanning electron microscopy (SEM) was carried out on a FEI Nova 400 Nano SEM and utilizing a through-the-lens (TLD) detector. The SEM images were acquired using a 32scan average.

2.4. Hall Measurement. The detection of preimmobilized SPM beads was achieved by employing an ac phase-sensitive technique as previously reported [45]. The Hall device was biased with a dc current $I=50 \mu \mathrm{A}$, and the beads were magnetized with an ac magnetic field; lock-in detection of the ac Hall voltage occurred at the magnetic field frequency. The application of an additional dc magnetic field reduced the SPM bead susceptibility and thus the ac magnetic field generated by the beads. This produced a drop in the ac Hall voltage signal indicating the presence of the beads.

\section{Results and Discussion}

3.1. Design. A schematic of the Hall magnetometer-based biosensor and detection strategy used for detection of a single-stranded DNA (ssDNA) target sequence by threestrand annealing over the surface of a $1 \mu \mathrm{m}^{2}$ Hall junction is shown in Figure 1. The biosensor platform is assembled in parallel steps to limit the processing time for target

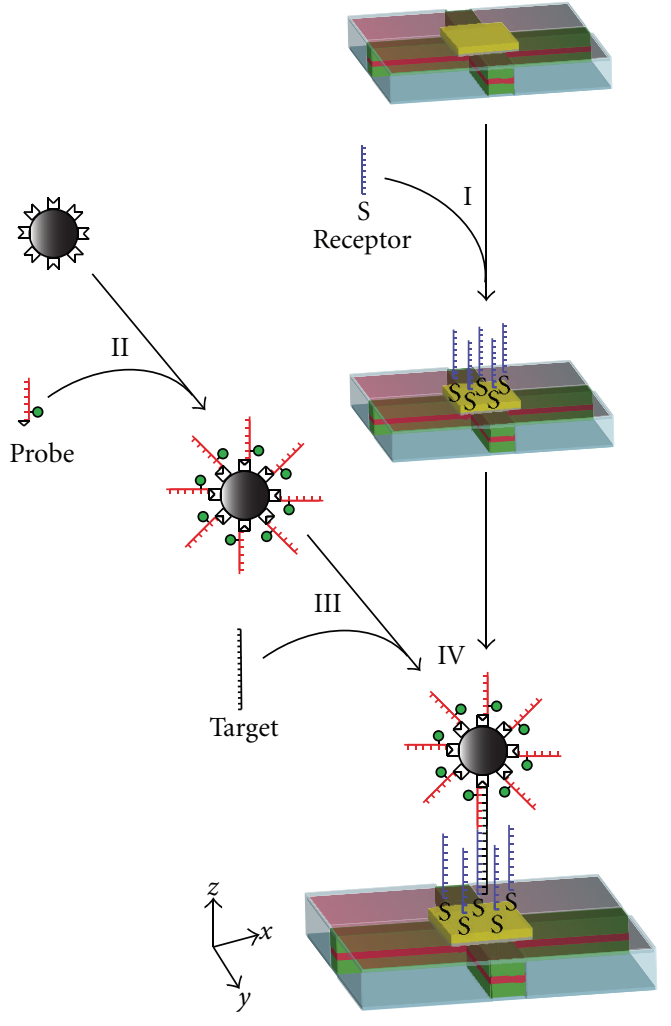

FIGURE 1: Generalized schematic for the detection of label-free target DNA using Hall magnetometry. The label-free target DNA (black) is detected by immobilization at the Hall device via complementary base pairing with receptor DNA (blue) preassembled on the Hall device surface to additional complementary probe DNA (red) with an internal fluorescent marker preconjugated to the surface of a magnetic nanobead resulting in a detectable Hall signal. Nanobead is not drawn to scale.

detection. This parallels work by others to detect threestrand annealing using different sensor modalities, SERS [29] and colorimetry-(gold plasmon shift) based technologies, by simultaneously annealing the target, sensor, and probe sequences [14, 26-28]. Our platform is composed of six $1 \mu \mathrm{m}^{2}$ Hall junctions (Figure 2(a)) etched into an epitaxially grown, vertically integrated InAs quantum well heterostructure isolated from the surrounding environment by a $60 \mathrm{~nm}$ overlayer of silicon dioxide, as previously described $[42,44]$. The six available Hall junctions are divided into a set of three bioactive sensors (i, ii, and iii) and three nonactive controls (ic, iic, and iiic). The bioactive sensors are generated via patterning $3 \mu \mathrm{m}$ gold bonding pads evaporated onto the $\mathrm{SiO}_{2}$ layer only over the bioactive junctions (i, ii, iii). The bonding pads provide a site for self-assembly of the receptor singlestrand DNA onto the surface of the Hall junction sensor without modifying the properties of the InAs quantum well heterostructures. The nonactive controls do not have the gold bonding pad. To minimize biofouling of the device by the biological constituents in the sample via nonspecific interactions, the exposed $\mathrm{SiO}_{2}$ surface is selectively modified by a polyethylene-glycol-conjugated silane moiety [49]. The bioactive junctions are modified by self-assembly of the 


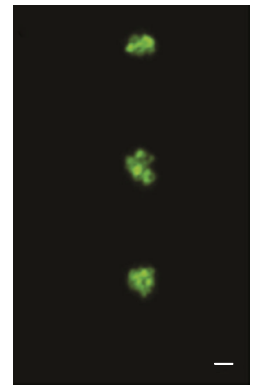

(a)

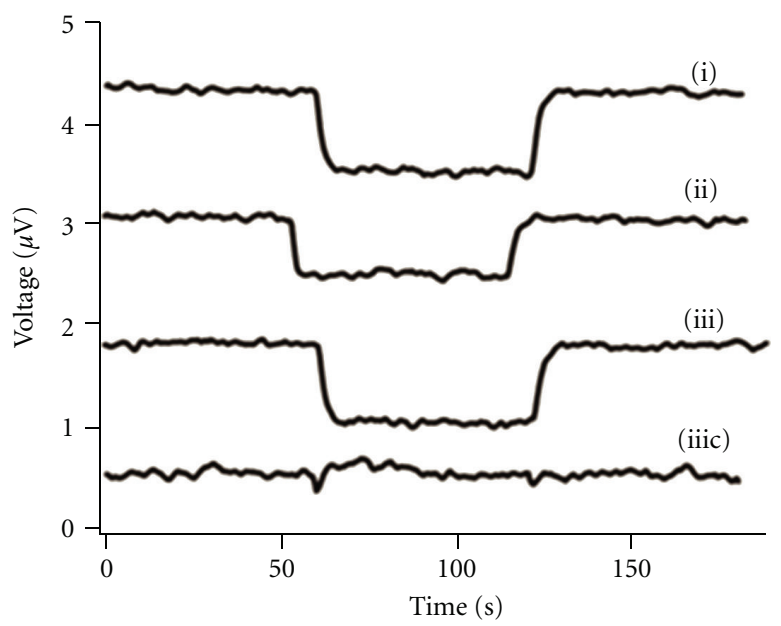

(c)

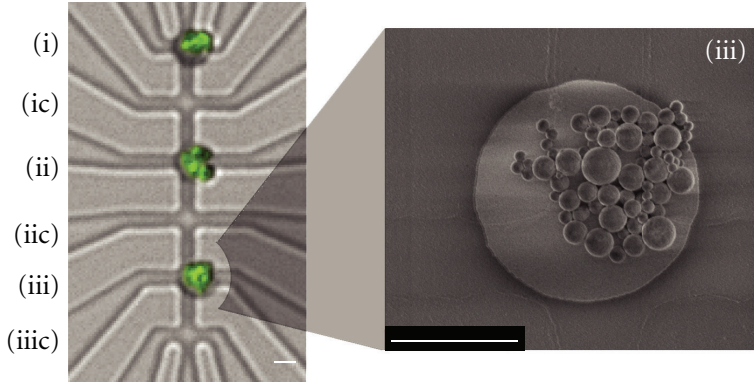

(b)



(d)

Figure 2: (a) Optical microscopy characterization (wide-field fluorescence and DIC overlay) of three-strand DNA assembly is shown by the presence of green fluorescence indicating the presence of probe DNA. (b) SEM was used to evaluate the location and to quantify the number of nanobeads contributing to the Hall response for (iii), where the grey box designates the location of the underlying Hall junction. (c) Hall responses for three active junctions (i, ii, iii) and a single control junction (iiic) are plotted as Hall voltage offset versus time; the presence of nanobeads over the active Hall junctions results in a drop in Hall voltage when a dc magnetic field is applied. (d) The theoretical device signal stemming from a single $344 \mathrm{~nm}$ SPM bead is shown to the right as a function of position over the Hall junction further illustrating the local sensitivity of Hall magnetometry. Scale bars $=2 \mu \mathrm{m}$ in (a) and (b).

receptor ssDNA (blue in Figure 1) onto the gold pads by exposure of a solution of the receptor to the Hall junction platform (Figure 1(I)) and subsequent washing to remove unbound DNA. The fluorescein-labeled probe ssDNA sequence (red in Figure 1) is preappended to the SPM bead platform via streptavidin-biotin conjugation (Figure 1-(II)).

Prior to the detection of the target DNA sequence (black in Figure 1), the probe strand and the target strand were prehybridized (Figure 1-(III)). The detection of the target DNA was then accomplished by annealing the SPM beadprobe-target complex with the receptor sequence (blue) preassembled at the surface of the Hall device platform at room temperature (Figure 1-(IV)). The assembly of the three-strand sequence requires $3 \mathrm{hrs}$, which is equivalent to standard FRET, plasmonic, GMR and SERS-based detection scenarios, but far faster than optical chip techniques that can require 16-24 hrs to achieve hybridization. Although the simultaneous addition of all three ssDNA components is experimentally feasible, stepwise assembly allowed the added benefit that the observed signal is not artificially enhanced by nonspecific, non-DNA bound SPM beads. All unbound nucleic acid species and nucleic acid-labeled SPM beads are removed by magnetic separation prior to final three-strand DNA assembly and washed prior to Hall detection. The strategy allows a specific binding event at the electrically isolated gold pad to induce a direct voltage response in the device without altering the device properties directly, as would be observed in SPR-based devices. The sequential, parallel assembly strategy (Figure 1(I)-(IV)) allows convenient concentration amplification for the target ssDNA from extraneous DNA fragments.

3.2. Detection. In Figure 2 the detection of the 35-base target on the dual optical/Hall device senor is shown for a $25 \mu \mathrm{L}$ droplet containing $7 \mu \mathrm{M}$ DNA. The specificity of the assembly of the three-strand DNA complex onto the gold pad (grey circle) over the Hall junction is clearly observed in the wide-field fluorescence overlaid with differential interference contrast (DIC) micrograph (Figure 2(a)). The observed green photoluminescence in Figure 2(a) arises from the fluorescein label on the probe strand and requires the three-strand annealing process to occur in order for the 
probe to be optically detectable. The lack of nonspecific binding of the probe to regions outside of the gold pad region confirms the specificity of the three-strand assembly protocol. The specificity of the assembly on the gold pads is further con-firmed by comparing the optical micrograph (Figure 2(a)) and a scanning electron micrograph of the same region (Figure 2(b); see Figure S1 in Supplementary Material available online at doi:10.1155/2012/492730). Scanning election microscopy (SEM) imaging of junction (iii) indicates that $\sim 73$ beads are present on the $3 \mu \mathrm{m}$ (diameter) gold pad. Inspection of the DIC image of junction (iii) (Figure 2(a)) reveals the registry between the underlying Hall junction in the SEM image (Figure 2(b), grey box), and the gold pad on the surface of the Hall magnetometer results in only $\sim 12$ na-nobeads being positioned directly or partially over the active area of the Hall junction.

For detection of DNA annealing, the presence of the SPM bead is measured as a change in voltage by the use of both ac and dc magnetic fields. The use of both ac and dc fields allows for a binding event signal to be cleanly isolated by using lock-in detection. In the absence of the external dc field, no signal is detectable in the Hall junction. The ac magnetic field of $3.76 \mathrm{mT}$ at $93 \mathrm{~Hz}$ is used to induce magnetization of the SPM nanobeads. The $70.6 \mathrm{mT} \mathrm{dc}$ magnetic field (NdFeB) is applied perpendicular to the Hall junction to shift the magnetization of the nanobeads to lower susceptibility as given by the expression $\Delta V_{H} \propto \Delta M$, where $\Delta M$ is the change in the ac magnetization before and after the dc field was applied. The Hall sensor was operated in constant current mode with an applied dc current of $50 \mu \mathrm{A}$.

The voltage responses to binding of the target sequence with the preappended probe and $350 \mathrm{~nm}$ magnetic beads to receptor strands on the three active junctions (i, ii, iii), plus one control junction without any beads (iiic) are shown in Figure 2(c). The voltage response for all junctions and controls are shown in Figure S1. The voltage across the control junction (iiic) is $0 \pm 0.03 \mu \mathrm{V}$. The successful assembly of ssDNA over the active Hall junctions results in a sharp drop in the measured Hall voltage for all three active sensor elements when a dc field was applied. The Hall voltage measurements are $0.79 \mu \mathrm{V}$ (signal/noise $(\mathrm{S} / \mathrm{N}) 40), 0.55 \mu \mathrm{V}$ (S/N 28), and $0.78 \mu \mathrm{V}(\mathrm{S} / \mathrm{N} 39)$, for (i, ii, iii), respectively. In Figure 2, the observed step function is generated by the application and removal of the external dc magnetic field in the presence of the small ac field to allow lock-in detection. The $\mathrm{S} / \mathrm{N}$ was determined by averaging the change in signal when the dc field was applied and dividing it by the average standard deviation from zero measured in the absence of the applied dc field. The standard deviation in measured signals for the active junctions is $0 \pm 0.02 \mu \mathrm{V}$ as shown in Figure S1.

To analyze the voltage change per binding event, the number of beads per Hall junction must be assessed. In Figure 2(a) and Figure S1, the SEM micrographs indicate the presence of 41 beads on junction (i), 68 beads on junction (ii), and 73 beads on junction (iii). In Figure 2(c), the voltage response for the three pads is similar regardless of the number of beads bound at the center of the Hall junction (Figure 2(c)). The largest expected voltage change in the Hall magnetometer will occur for beads directly over the Hall junction, falling rapidly for beads positioned $>0.5 \mu \mathrm{m}$ from the device center (Figure $2(\mathrm{~d})$ ). Directly over the Hall junction (shaded region Figure 2(b)), junction (i) has 8 beads, junction (ii) has 11 beads, and junction (iii) has 12 beads. Calculation of the predicted voltage response as a function of the distance of the bead from the center of the Hall junction is shown in Figure 2(d). For a single $350 \mathrm{~nm}$ magnetic bead approximately $272 \mathrm{~nm}$ from the Hall device, a voltage response of $0.4 \mu \mathrm{V}$ per bead is expected. The experimental value of $\sim 0.6-0.8 \mu \mathrm{V}$ measured in Figure 2(c) following DNA annealing of the target and probe indicates that more than one bead but not all of the bound beads contribute to the measured Hall voltage. For junction (iii), the result suggests the measured voltage is likely dominated by the 12 beads directly over the Hall junction (only beads contained within the grey box in Figure 2(b)). Due to the large size dispersion and subsequent large magnetic content variability in the commercially obtained SPM beads utilized in this study, the calculation of the number of beads contributing to the measured signal cannot be obtained if more than one bead lies directly over the underlying Hall junction. Based on the theoretical voltage (Figure 2(d)) and the observed voltage in response to DNA annealing (Figure 2(c)), a single bead should be detectable.

3.3. Single-Bead Detection. Although magnetic transduction devices are remarkably sensitive with detection of a single bead $(<500 \mathrm{~nm})$ reported for an antibody-antigen sandwichassay-based assembly of a magnetic bead on a micron-sized Hall device [44], and the multiple-bead detection by GMR devices [36], the report of three-stand DNA target detection at the single-magnetic-bead level by a Hall device has not been reported to date. Single-bead detection was observed for a 35-base pair DNA annealing event onto a Hall device, as shown in Figure 3. The Hall voltage response (Figure 3(b)) and corresponding SEM image (Figure 3(c)) for the twostrand annealing event (Figure 3(a)) indicate a voltage of 0.34 $\pm 0.03 \mu \mathrm{V}(0 \pm 0.04 \mu \mathrm{V}$ for control junction) for the two observed beads near the Hall junction. The two-dimensional (2D) theoretical Hall response has recently been modeled with respect to SPM bead position over the Hall junction [50]. The theoretical response for a single bead over the Hall junction in Figure 3 is shown in Figure 3(d), where red indicates a SPM particle positioned at the center of the device, while blue is a SPM outside the detectable range of the Hall junction. The measured voltage in Figure 3 is consistent with the theoretical value for a single bead, and therefore it is believed that the measured Hall voltage reflects only one of the two beads, since only one of the beads lies within the red zone for the theoretical plot (arrow in Figure 3(c)). Improvement of the signal-to-noise ratio can be achieved by operating the Hall device at higher frequencies; however, it is important to note that the sensitivity of the device can clearly distinguish a single-bead binding event from the noise floor by an order of magnitude at the frequency utilized in this study. 


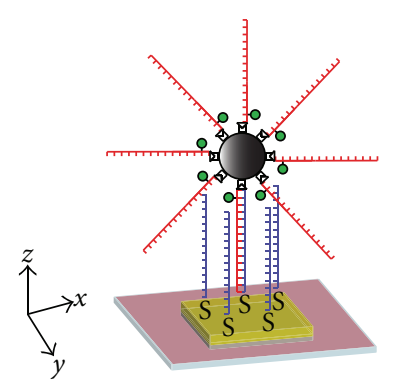

(a)

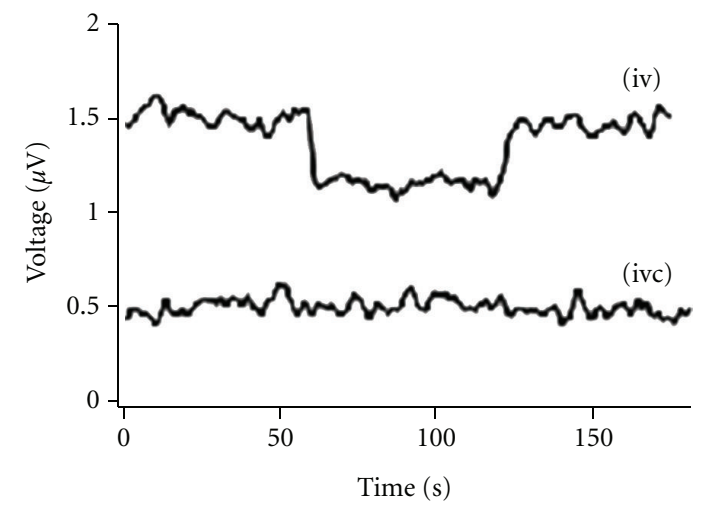

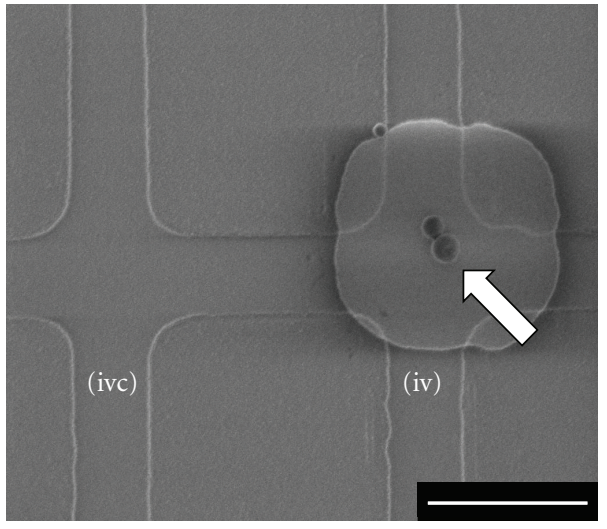

(c)

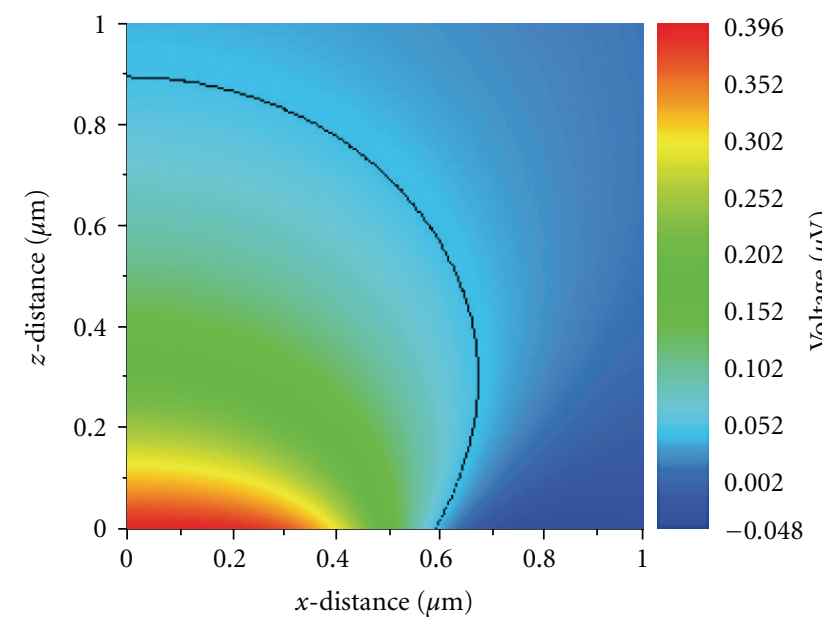

(d)

FIgURE 3: Sequence-specific two-strand DNA assembly and subsequent Hall detection of a single $344 \mathrm{~nm}$ nanobead. (a) Pictorial representation of two-strand DNA assembly, where the probe DNA (red) is complementary to the receptor DNA (blue). (b) Hall response data for the active (iv) and control Hall junction (ivc) plotted as Hall voltage versus time, where the drop in Hall voltage corresponds to the presence of a magnetic nanobead. (c) SEM was used to confirm that only one nanobead contributed significantly to the signal measured in (iv); scale bar $=2 \mu \mathrm{m}$. (d) The theoretical Hall device cross-sectional response for a single $344 \mathrm{~nm}$ SPM bead as a function of position from the center of the junction, where red indicates strongest change in voltage, aqua indicates weakest voltage change, and blue indicates a negative voltage readout. The noise floor for the device is outlined in black for reference.

3.4. Selectivity and Detection Limits. The limit of detection for DNA in a real sample will reflect the length and sequence of target DNA (both of which influence annealing temperature), and the concentration of DNA, and the concentration of DNA present in a milieu of nontarget DNA. Since sensitivity will be influenced by bead size and the area of the transduction platform, the concentration limit of detection for target DNA was assessed using optical microscopy analysis of the binding of the target DNA onto $2 \times 4 \mu \mathrm{m}$ gold patterns that serve as mimics of the GaAs Hall devices.

The thermodynamic stability of the three-strand DNA approach has been used for several sensor approaches, including optical, SERS, and colorimetric platforms. In the current study the stability of the three strands was experimentally verified using a gel shift assay (Figure S3). In Figures $4(\mathrm{a})$ and $4(\mathrm{~b})$, binding of the 35-base pair three-strand DNA 


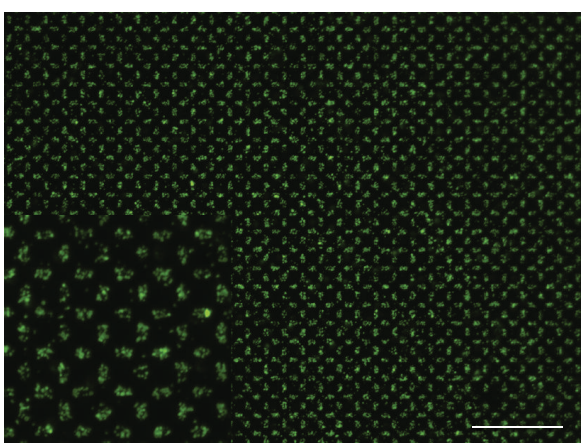

(a)

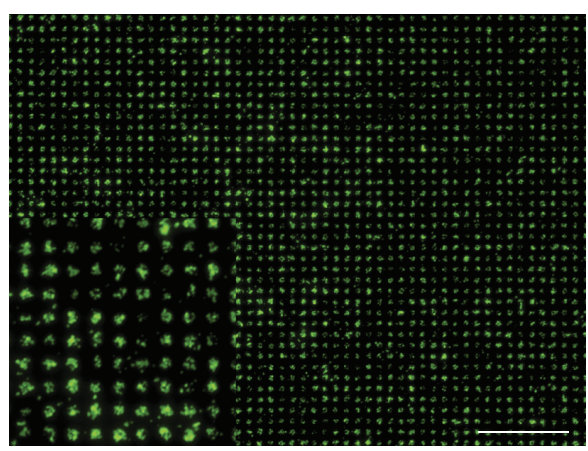

(b)

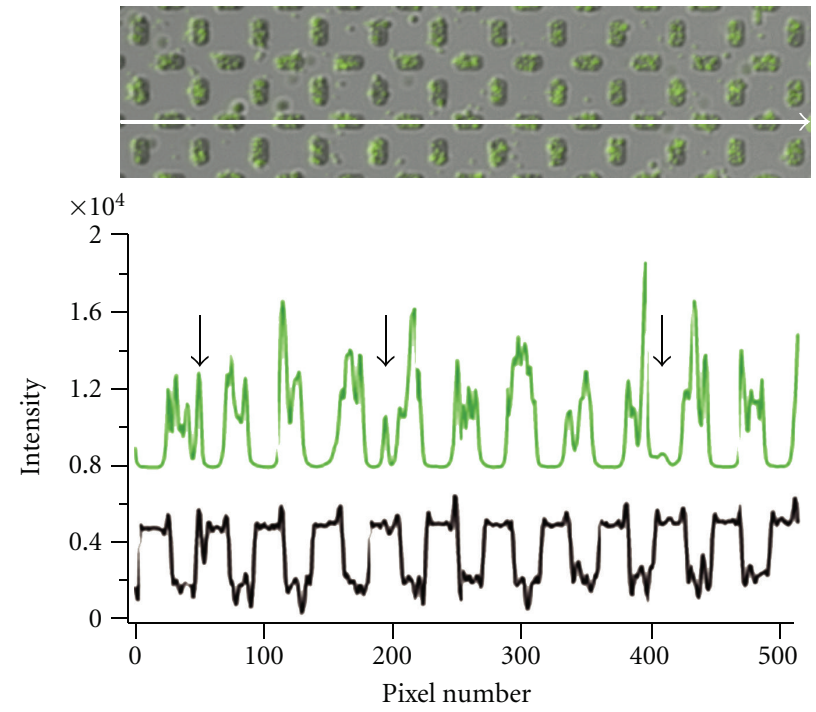

(c)

Figure 4: Three-strand DNA assembly on a mimic array (patterned on a GaAs substrate) for (a) complementary target only and (c) 10 ppm target in nontarget DNA. The inlays in the lower left of (a) and (c) are an enlarged portion of (a) and (c), respectively. Scale bars $=50 \mu \mathrm{m}$. (b) A line scan of the wide-field fluorescence microscopy image in (a) showing fluorescein-labeled probe DNA (green) and DIC (black) intensity correlates fluorescence intensity with nanobeads located primarily over gold pads, where the black arrows signify the presence of a small number of nonspecifically bound nanobeads.

assembly onto a mimic was assessed by the fluorescently labeled probe sequence conjugated to the $350 \mathrm{~nm}$ magnetic beads. Inspection of a line scan for the fluorescence intensity from the probe sequence shows good correlation with the Hall mimic patterns (Figure 4(b)). The signal fluctuations do not indicate single-bead response as the fluorescein intensity depends on the particle size, number of DNA probe strands, labeling efficiency, and focal plane of the microscopy image. Little intensity is observed over the control PEGylated regions surrounding gold pads (identified with black arrows). The discrimination level is $>10,000$ counts above background for selective target DNA binding at the gold pads in buffered solution (Figure 4(a) and Figure S2).

An important measure of device performance is the ability to discriminate target ssDNA in the presence of extraneous (noncomplementary) sequences in solution, particularly at low levels of target DNA. The ability to discriminate target DNA in the presence of nontarget sequences was analyzed by optical microscopy on $3 \mu \mathrm{m}$ patterned GaAs Hall device mimics in a buffered solution. Since the sensitivity of the device was demonstrated to achieve a limit of detection that is consistent with a single bead $(0.34 \mu \mathrm{V}$ versus $0 \pm 0.04 \mu \mathrm{V}$ noise floor), the choice of an optical mimic to only probe fidelity over a Hall junction allows analysis of the limit of detection for the three-strand annealing process. Fluorescently tagged nanobeads were selectively annealed at gold pads at a concentration of $364 \mathrm{pM}$ target DNA in a solution containing $36 \mu \mathrm{M}$ nontarget DNA, which corresponds to less than two complementary target DNA sequences per $350 \mathrm{~nm}$ nanobead (Figure 4(c)). The measurements equate to detection at the $10 \mathrm{ppm}$ level target. For comparison, a mimic array in which the receptor strand was noncomplementary to the target strand clearly demonstrated that nonspecific DNA binding is not observed (Figure S2). Although we have not yet tested cellular extracts of nucleic acids, the sensitivity and selectivity of the device-detection of a single SPM bead at a Hall junction, corresponding to 1-2 target DNA moleculesclearly demonstrate for the first time that this technology holds substantial promise for biomoleculesensing.

\section{Conclusion}

The device strategy utilizing three-strand DNA assembly on a Hall magnetometer provides a detection platform with high specificity, low limit of detection (single SPM bead, and small numbers of target DNA molecules), and very high fidelity. Sensitivity of the Hall biosensor is attributable to the properties of the Hall junction and is dependent on the size of the Hall junction, the frequency of the ac field oscillation, the moment of the SPM bead, and the distance of the SPM bead from the Hall junction. In the nanotechnology device, 
the use of a SPM nanobead does not hinder the specificity of Watson-Crick base pairing for the target nucleic acid as evidenced by sequence-specific DNA hybridization (Figure 2 and Figure S2). At the detection frequency employed in this study, the 3D plot in Figure 3(d) indicates the possibility of detecting an SPM bead at distances approaching $0.9 \mu \mathrm{m}$ off the surface of the device when the SPM bead is located directly over the center of the Hall junction, which may allow much longer sequences of DNA to be detected. Higher frequency measurements will decrease the noise level and therefore increase the sensitivity of the device to the magnetic bead position.

We have demonstrated the successful use of Hall magnetometry to detect a 35-base target DNA at the singlemagnetic-bead level that could be applied for POC diagnostics. Reduction of the dimension of the gold pad and improved registry, as well as bead homogeneity could be used to further improve upon the overall device performance. Extrapolation of the device to a microarray of selectively labeled Hall sensors could represent a transformative biosensor platform. The parallel Hall device strategy could allow multiple DNA sequences to be simultaneously detected in a biological matrix since each magnetic bead and probe strand can be bar-coded by dye photoluminescence [23] and SPM bead size since the response will be proportional to the SPM moment. Alternatively, the receptor DNA on the Hall junction can be selectively dip-penned for multisequence analysis [51]. By eliminating concerns associated with sample amplification [3] such an array would allow screening for nucleic acid targets of biomedical interest such as pathogens or disease-related mutations [52-56].

\section{Acknowledgments}

Support was provided by NIH grant GM079592. The authors thank Kimberly A. Riddle and Thomas J. Fellers in the Florida State University's Biological Science Imaging Resource (BSIR) for extensive SEM characterization and Eric J. Lochner in the Center of Materials Research and Technology (MARTECH) at FSU for material characterization.

\section{References}

[1] D. V. Lim, J. M. Simpson, E. A. Kearns, and M. F. Kramer, "Current and developing technologies for monitoring agents of bioterrorism and biowarfare," Clinical Microbiology Reviews, vol. 18, no. 4, pp. 583-607, 2005.

[2] D. Ivnitski, D. J. O’Neil, A. Gattuso, R. Schlicht, M. Calidonna, and R. Fisher, "Nucleic acid approaches for detection and identification of biological warfare and infectious disease agents," BioTechniques, vol. 35, no. 4, pp. 862-869, 2003.

[3] J. Ince and A. McNally, "Development of rapid, automated diagnostics for infectious disease: advances and challenges," Expert Review of Medical Devices, vol. 6, no. 6, pp. 641-651, 2009.

[4] D. A. Giljohann and C. A. Mirkin, "Drivers of biodiagnostic development," Nature, vol. 462, no. 7272, pp. 461-464, 2009.

[5] O. Lazcka, F. J. D. Campo, and F. X. Muñoz, "Pathogen detection: a perspective of traditional methods and biosensors," Biosensors \& Bioelectronics, vol. 22, no. 7, pp. 1205-1217, 2007.
[6] J. Wang, "Electrochemical biosensors: towards point-of-care cancer diagnostics," Biosensors \& Bioelectronics, vol. 21, no. 10, pp. 1887-1892, 2006.

[7] T. M. Herne and M. J. Tarlov, "Characterization of DNA probes immobilized on gold surfaces," Journal of the American Chemical Society, vol. 119, no. 38, pp. 8916-8920, 1997.

[8] T. Wink, S. J. Van Zuilen, A. Bult, and W. P. Van Bennekom, "Self-assembled monolayers for biosensors," Analyst, vol. 122, no. 4, pp. R43-R50, 1997.

[9] G. Festag, A. Steinbrück, A. Wolff, A. Csaki, R. Möller, and W. Fritzsche, "Optimization of gold nanoparticle-based DNA detection for microarrays," Journal of Fluorescence, vol. 15, no. 2, pp. 161-170, 2005.

[10] X. D. Song, J. Shi, and B. Swanson, "Flow cytometry-based biosensor for detection of multivalent proteins," Analytical Biochemistry, vol. 284, no. 1, pp. 35-41, 2000.

[11] L. Shi, L. H. Reid, W. D. Jones et al., "The MicroArray Quality Control (MAQC) project shows inter- and intraplatform reproducibility of gene expression measurements," Nature Biotechnology, vol. 24, no. 9, pp. 1151-1161, 2006.

[12] Y. W. C. Cao, R. Jin, and C. A. Mirkin, "Nanoparticles with Raman spectroscopic fingerprints for DNA and RNA detection," Science, vol. 297, no. 5586, pp. 1536-1540, 2002.

[13] C. T. Campbell and G. Kim, "SPR microscopy and its applications to high-throughput analyses of biomolecular binding events and their kinetics," Biomaterials, vol. 28, no. 15, pp. 2380-2392, 2007.

[14] T. A. Taton, C. A. Mirkin, and R. L. Letsinger, "Scanometric DNA array detection with nanoparticle probes," Science, vol. 289, no. 5485, pp. 1757-1760, 2000.

[15] B. Dubertret, M. Calame, and A. J. Libchaber, "Singlemismatch detection using gold-quenched fluorescent oligonucleotid," Nature Biotechnology, vol. 19, no. 4, pp. 365-370, 2001.

[16] W. R. Algar, M. Massey, and U. J. Krull, "The application of quantum dots, gold nanoparticles and molecular switches to optical nucleic-acid diagnostics," TrAC Trends in Analytical Chemistry, vol. 28, no. 3, pp. 292-306, 2009.

[17] S. Husale, H. H. J. Persson, and O. Sahin, "DNA nanomechanics allows direct digital detection of complementary DNA and microRNA targets," Nature, vol. 462, no. 7276, pp. 1075-1078, 2009.

[18] N. G. Clack, K. Salaita, and J. T. Groves, "Electrostatic readout of DNA microarrays with charged microspheres," Nature Biotechnology, vol. 26, no. 7, pp. 825-830, 2008.

[19] D. S. Johnson, W. Li, D. B. Gordon et al., "Systematic evaluation of variability in ChIP-chip experiments using predefined DNA targets," Genome Research, vol. 18, no. 3, pp. 393-403, 2008.

[20] W. R. Algar and U. J. Krull, "Toward a multiplexed solidphase nucleic acid hybridization assay using quantum dots as donors in fluorescence resonance energy transfer," Analytical Chemistry, vol. 81, no. 10, pp. 4113-4120, 2009.

[21] B. S. Gaylord, A. J. Heeger, and G. C. Bazan, "DNA detection using water-soluble conjugated polymers and peptide nucleic acid probes," Proceedings of the National Academy of Sciences of the United States of America, vol. 99, no. 17, pp. 10954-10957, 2002.

[22] J. Zhang, B. P. Ting, N. R. Jana, Z. Gao, and J. Y. Ying, "Ultrasensitive electrochemical DNA biosensors based on the detection of a highly characteristic solid-state process," Small, vol. 5, no. 12, pp. 1414-1417, 2009. 
[23] Y. Li, Y. T. H. Cu, and D. Luo, "Multiplexed detection of pathogen DNA with DNA-based fluorescence nanobarcodes," Nature Biotechnology, vol. 23, no. 7, pp. 885-889, 2005.

[24] W. J. Qin, O. S. Yim, P. S. Lai, and L. Y. L. Yung, "Dimeric gold nanoparticle assembly for detection and discrimination of single nucleotide mutation in Duchenne muscular dystrophy," Biosensors \& Bioelectronics, vol. 25, no. 9, pp. 2021-2025, 2010.

[25] V. C. Martins, F. A. Cardoso, J. Germano et al., "Femtomolar limit of detection with a magnetoresistive biochip," Biosensors \& Bioelectronics, vol. 24, no. 8, pp. 2690-2695, 2009.

[26] C. A. Mirkin, R. L. Letsinger, R. C. Mucic, and J. J. Storhoff, "A DNA-based method for rationally assembling nanoparticles into macroscopic materials," Nature, vol. 382, no. 6592, pp. 607-609, 1996.

[27] J. J. Storhoff, S. S. Marla, P. Bao et al., "Gold nanoparticlebased detection of genomic DNA targets on microarrays using a novel optical detection system," Biosensors \& Bioelectronics, vol. 19, no. 8, pp. 875-883, 2004.

[28] J. J. Storhoff, A. D. Lucas, V. Garimella, Y. P. Bao, and U. R. Müller, "Homogeneous detection of unamplified genomic DNA sequences based on colorimetric scatter of gold nanoparticle probes," Nature Biotechnology, vol. 22, no. 7, pp. 883-887, 2004.

[29] G. Braun, S. J. Lee, M. Dante, T. Q. Nguyen, M. Moskovits, and N. Reich, "Surface-enhanced raman spectroscopy for DNA detection by nanoparticle assembly onto smooth metal films," Journal of the American Chemical Society, vol. 129, no. 20, pp. 6378-6379, 2007.

[30] S. P. Mulvaney, C. L. Cole, M. D. Kniller et al., "Rapid, femtomolar bioassays in complex matrices combining microfluidics and magnetoelectronics," Biosensors \& Bioelectronics, vol. 23, no. 2, pp. 191-200, 2007.

[31] D. L. Arruda, W. C. Wilson, C. Nguyen et al., "Microelectrical sensors as emerging platforms for protein biomarker detection in point-of-care diagnostics," Expert Review of Molecular Diagnostics, vol. 9, no. 7, pp. 749-755, 2009.

[32] J. Schotter, P. B. Kamp, A. Becker, A. Pühler, G. Reiss, and H. Brückl, "Comparison of a prototype magnetoresistive biosensor to standard fluorescent DNA detection," Biosensors \& Bioelectronics, vol. 19, no. 10, pp. 1149-1156, 2004.

[33] J. Germano, V. C. Martins, F. A. Cardoso et al., "A portable and autonomous magnetic detection platform for biosensing," Sensors, vol. 9, no. 6, pp. 4119-4137, 2009.

[34] D. L. Graham, H. A. Ferreira, N. Feliciano, P. P. Freitas, L. A. Clarke, and M. D. Amaral, "Magnetic field-assisted DNA hybridisation and simultaneous detection using micron-sized spin-valve sensors and magnetic nanoparticles," Sensors and Actuators B, vol. 107, no. 2, pp. 936-944, 2005.

[35] H. A. Ferreira, D. L. Graham, N. Feliciano, L. A. Clarke, M. D. Amaral, and P. P. Freitas, "Detection of cystic fibrosis related DNA targets using AC field focusing of magnetic labels and spin-valve sensors," IEEE Transactions on Magnetics, vol. 41, no. 10, pp. 4140-4142, 2005.

[36] D. A. Hall, R. S. Gaster, T. Lin et al., "GMR biosensor arrays: a system perspective," Biosensors \& Bioelectronics, vol. 25, no. 9, pp. 2051-2057, 2010.

[37] S. J. Osterfeld, H. Yu, R. S. Gaster et al., "Multiplex protein assays based on real-time magnetic nanotag sensing," Proceedings of the National Academy of Sciences of the United States of America, vol. 105, no. 52, pp. 20637-20640, 2008.

[38] D. R. Baselt, G. U. Lee, M. Natesan, S. W. Metzger, P. E. Sheehan, and R. J. Colton, "A biosensor based on magnetoresistance technology," Biosensors \& Bioelectronics, vol. 13, no. 7-8, pp. 731-739, 1998.
[39] R. L. Edelstein, C. R. Tamanaha, P. E. Sheehan et al., "The BARC biosensor applied to the detection of biological warfare agents," Biosensors \& Bioelectronics, vol. 14, no. 10-11, pp. 805$813,2000$.

[40] Y. Li, P. Xiong, S. Von Molnár, S. Wirth, Y. Ohno, and H. Ohno, "Hall magnetometry on a single iron nanoparticle," Applied Physics Letters, vol. 80, no. 24, pp. 4644-4646, 2002.

[41] A. Sandhu, Y. Kumagai, A. Lapicki, S. Sakamoto, M. Abe, and H. Handa, "High efficiency Hall effect micro-biosensor platform for detection of magnetically labeled biomolecules," Biosensors \& Bioelectronics, vol. 22, no. 9-10, pp. 2115-2120, 2007.

[42] G. Mihajlović, P. Xiong, S. von Molnár et al., "Detection of single magnetic bead for biological applications using an InAs quantum-well micro-Hall sensor," Applied Physics Letters, vol. 87, no. 11, Article ID 112502, 3 pages, 2005.

[43] G. Mihajlović, P. Xiong, S. von Molnár, M. Field, and G. J. Sullivan, "InAs quantum well Hall devices for roomtemperature detection of single magnetic biomolecular labels," Journal of Applied Physics, vol. 102, no. 3, Article ID 034506, 9 pages, 2007.

[44] P. Manandhar, K. S. Chen, K. Aledealat et al., "The detection of specific biomolecular interactions with micro-Hall magnetic sensors," Nanotechnology, vol. 20, no. 35, Article ID 355501, 2009.

[45] P. A. Besse, G. Boero, M. Demierre, V. Pott, and R. Popovic, "Detection of a single magnetic microbead using a miniaturized silicon Hall sensor," Applied Physics Letters, vol. 80, no. 22, Article ID 4199, 3 pages, 2002.

[46] R. S. Gaster, D. A. Hall, C. H. Nielsen et al., "Matrix-insensitive protein assays push the limits of biosensors in medicine," Nature Medicine, vol. 15, no. 11, pp. 1327-1332, 2009.

[47] G. Mihajlovic and S. von Molnar, in Nanoscale Magnetic Materials and Applications, J. P. Liu, E. Fullerton, O. Gutfleisch, and D. J. Sellmyer, Eds., pp. 685-710, Springer, New York, NY, USA, 2009.

[48] M. Behet, J. Bekaert, J. De Boeck, and G. Borghs, "InAs/ Al0.2Ga0.8Sb quantum well Hall effect sensors," Sensors and Actuators A, vol. 81, no. 1-3, pp. 13-17, 2000.

[49] B. Kannan, R. P. Kulkarni, and A. Majumdar, "DNA-based programmed assembly of gold nanoparticles on lithographic patterns with extraordinary specificity," Nano Letters, vol. 4, no. 8, pp. 1521-1524, 2004.

[50] K. Aledealat, G. Mihajlović, K. Chen et al., "Dynamic microhall detection of superparamagnetic beads in a microfluidic channel," Journal of Magnetism and Magnetic Materials, vol. 322, no. 24, pp. L69-L72, 2010.

[51] L. M. Demers, D. S. Ginger, S. J. Park, Z. Li, S. W. Chung, and C. A. Mirkin, "Direct patterning of modified oligonucleotides on metals and insulators by dip-pen nanolithography," Science, vol. 296, no. 5574, pp. 1836-1838, 2002.

[52] J. Köhler, Y. Chen, B. Brenner et al., "Familial hypertrophic

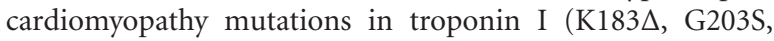
K206Q) enhance filament sliding," Physiological Genomics, vol. 14, pp. 117-128, 2003.

[53] M. S. Parmacek and R. J. Solaro, "Biology of the troponin complex in cardiac myocytes," Progress in Cardiovascular Diseases, vol. 47, no. 3, pp. 159-176, 2004.

[54] B. Gafurov, S. Fredricksen, A. Cai, B. Brenner, P. B. Chase, and J. M. Chalovich, "The $\Delta 14$ mutation of human cardiac troponin T enhances ATPase activity and alters the cooperative binding of S1-ADP to regulated actin," Biochemistry, vol. 43, no. 48, pp. 15276-15285, 2004. 
[55] F. Bai, A. Weis, A. K. Takeda, P. B. Chase, and M. Kawai, "Enhanced active cross-bridges during diastole: molecular pathogenesis of tropomyosin's HCM mutations," Biophysical Journal, vol. 100, no. 4, pp. 1014-1023, 2011.

[56] M. C. Mathur, P. B. Chase, and J. M. Chalovich, "Several cardiomyopathy causing mutations on tropomyosin either destabilize the active state of actomyosin or alter the binding properties of tropomyosin," Biochemical and Biophysical Research Communications, vol. 406, no. 1, pp. 74-78, 2011. 

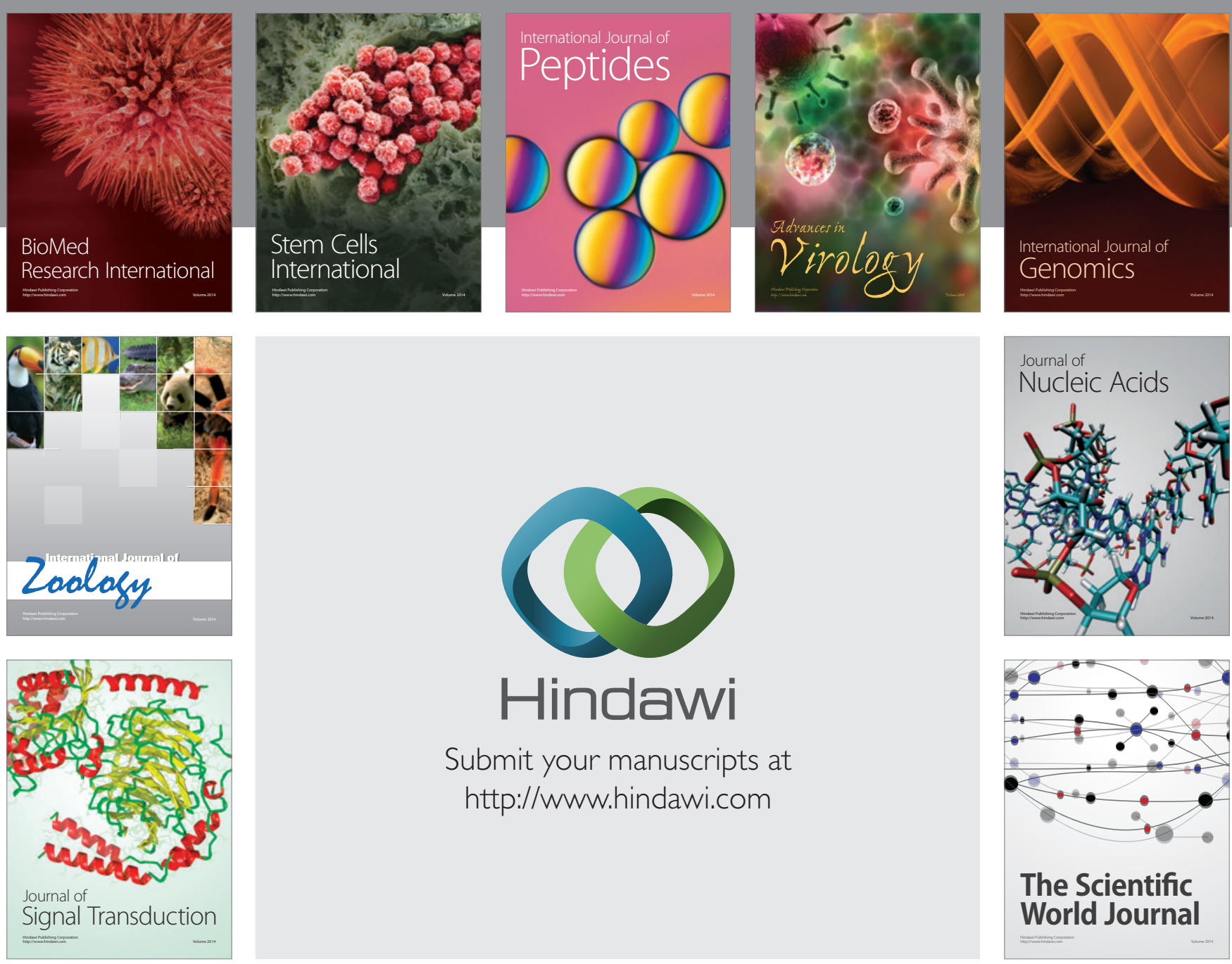

Submit your manuscripts at

http://www.hindawi.com
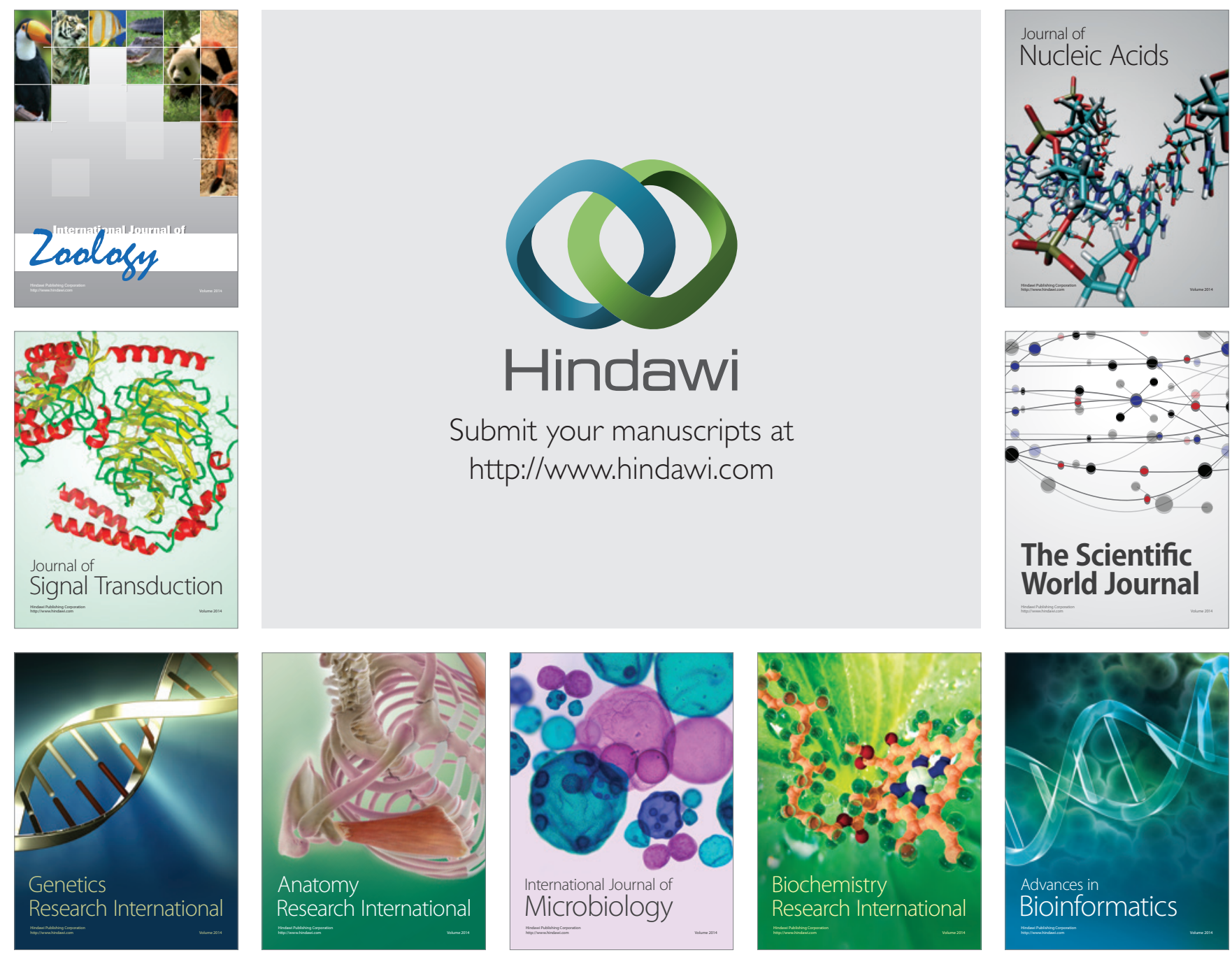

The Scientific World Journal
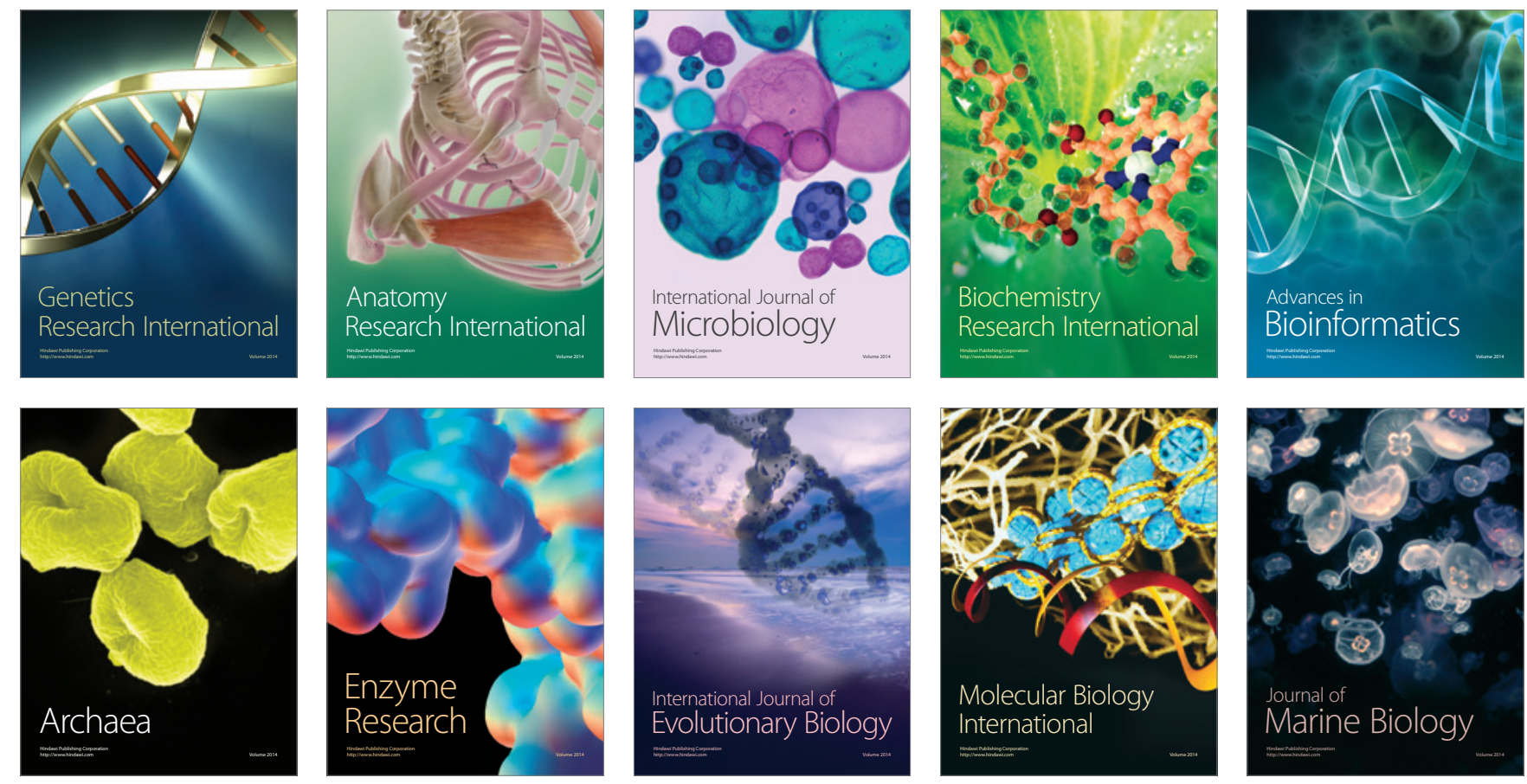